\title{
Spectrum of surface-mode contributions to the excitation probability for electron beam interacting with sharp-edged dielectric wedges
}

\author{
H.B.Nersisyan and A.V.Hovhannisyan \\ Institute of Radiophysics and Electronics, \\ Ashtarak-2, 378410, Armenia
}

May 15, 2017

\begin{abstract}
The interaction of a nonrelativistic charged particle beam, travelling parallel to the surface of a sharp-edged dielectric wedge is analyzed. The general expressions for excitation probability are obtained for a beam moving along the direction of a symmetry axis, either outside or inside the dielectric wedge. The dielectric function of the medium is assumed to be isotropic, and numerical results are given for the materials of experimental interest.
\end{abstract}

\section{Introduction}

The tradition of analyzing material targets from the energy-loss spectroscopy of charged particles scattered through or near a scatter has continued to enrich physics over the past several decades. Applications have been found in nuclear and particle physics, atomic and molecular physics, and in condensed-matter physics. Recently, biological physics has benefited as well, particularly from the use of electron-energy-loss spectroscopy (EELS) performed using scanningtransmission-electron microscopes (STEM). Using a STEM one can obtain information on the size, shape, composition, and location of isolated particulates embedded in a host material (composite) and thus obtain three-dimensional chemical maps with high resolution (Chen et al 1986).

In a typical STEM configuration, a well-focused $0.5-\mathrm{nm}$ probe of $50-100 \mathrm{keV}$ electrons provides a high-resolution transmission scanning image for samples with complex structures. It also yields from selected regions of the structure, x-ray emission spectra and electron energy-loss spectra. Quantitative theories have 
been developed to analyze the experimental energy-loss spectra in some simple cases.

Solutions, within the classical dielectric theory, have been worked out for a number of cases involving planar interfaces (Echenique and Pendry 1975), spheres (Ferrel and Echenique 1985, Echenique et al 1987), cylinders (Walsh 1989, Zabala et al 1989), spheroids (Illman et al 1988), and parabolically shaped wedges (Garcia-Molina et al 1985). For these simple geometries, experimental results show that dielectric excitation theory is capable of predicting energy-loss spectra, allowing a fully consistent dielectric characterization of an interface or a small particle (Walls and Howie 1989, Rivacoba et al 1992).

In this paper we focus our attention on the calculation of excitation probability for point charge particle moving parallel to the sharp-edged dielectric wedge whose boundary is formed by the intersection of two semi-infinite planes making an interior angle of $\alpha$. Our interest is explained by some experiments that were reported in (Marks 1982, Cowley 1982, Wheatley et al 1983). The targets $\left(\mathrm{MgO}, \mathrm{NiO}, \mathrm{Al}_{2} \mathrm{O}_{3}\right.$, etc.) bombarded in the experiments are of cubic symmetry (i.e. have sharp-edged form), about 20-200 $\mathrm{nm}$ in size, and the electron beam is oriented along the principal crystallographic directions. Marks (1982) have measured the spectra of energy-losses of electron beams interacting with small crystallites of $\mathrm{MgO}$. The calculations for excitation probability were done considering the crystallites as semi-infinite surface related to electron beams. Using the classical theory of impact-parameter-dependent energy losses for planar interfaces Wheatley et al (1983) applied the planar results for spheres while resolving the force in the track direction. Garcia-Molina et al (1985) calculated the energy-loss function and the excitation probability of the wedge modes due to a pointlike electron beam moving parallel to the dielectric wedge surface with a parabolic-cylinder boundary.

Here we shall calculate the excitation probability of sharp-edged modes, due to an electron beam passing parallel to the dielectric wedge with a local, but otherwise arbitrary, dielectric function $\varepsilon(\omega)$. We shall limit our calculations to the nonretarded limit. The limiting cases for the potential and excitation probability are shown. First, from exact expressions for excitation probability we derive the expressions for planar geometry in the limiting case of $\alpha=\pi$. Second, in Appendix we also show that in the static limit $(u \rightarrow 0$, where $u$ is the velocity of particle) from our results for the potential follows the expression for conducting sharp-edged wedge $(\varepsilon \rightarrow \infty)$ (Landau and Lifshitz 1982). In Sec. III we have utilized available bulk optical data (Roessler and Walker 1967) to calculate the differential energy-loss probability for $\mathrm{MgO}$. The theoretical predictions are compared with experimental data, and it is shown that the main features of the experiments reported by Marks (1982) can be explained by the theory. Also, our results are compared with a parabolically shaped wedge (Garcia-Molina et al 1985). In Sec. IV we present our conclusions and comments on the results and discuss opportunities for possible future work. 


\section{$2 \quad$ Energy loss and excitation probability}

Take dielectric wedges whose boundaries are formed by the intersection of two semi-infinite planes making an interior angle of $\alpha$ infinite in the $z$ direction. Let in the cylindrical system of coordinates $\rho, \theta, z$ the azymutal angle $\theta$ is measured from one of wedges sides. We consider two media filling the spaces $-\infty<z<+\infty$, $0 \leq \theta \leq \alpha, \alpha \leq \theta \leq 2 \pi$, and characterized by an isotropic dielectric functions $\varepsilon_{1}(\omega)$ and $\varepsilon_{2}(\omega)$ respectively (see figure 1 ). The incident charged particle with the charge $q$ moves with a velocity $u$ directed along the $z$ axis and has the following coordinates $\rho=a, \theta=\gamma(\gamma<\alpha), z=u t$.

The study of the electrostatic edge modes along a sharp-edged wedge is due to Dobrzynsky and Maradudin (1972), who solved Laplace's equation in the appropriate coordinate system. Davis (1976) has considered the electrostatic modes of a hyperbolic cylinder and has concluded that the results in the work of Dobrzynsky and Maradudin (1972) are associated with the sharpness of the edge of the wedge. Here we shall only give the main steps in the derivation of the electrostatic potential originated as a result of an electron beam traveling along the wedges surfaces.

We solve Poisson's equation for the potential

$$
\nabla^{2}(\widehat{\varepsilon} \varphi)=-\frac{4 \pi q}{a} \delta(\rho-a) \delta(\theta-\gamma) \delta(z-u t)
$$

where the charge density associated with an electron beam, which is described classically by a $\delta$ functions, $\widehat{\varepsilon}$ is the operator of dielectric permeability of the medium (Landau and Lifshitz 1982).

It is convenient to work in Fourier space:

$$
\varphi(\rho, \theta, \xi)=\int_{-\infty}^{+\infty} d \omega \exp (i \omega \xi / u) \varphi_{\omega}(\rho, \theta)
$$

where $\xi=z-u t$. Then, Poisson's equation becomes, in cylindrical coordinates, with $\varphi_{\omega}(\rho, \theta)$,

$$
\left(\frac{\partial^{2}}{\partial \rho^{2}}+\frac{1}{\rho} \frac{\partial}{\partial \rho}+\frac{1}{\rho^{2}} \frac{\partial^{2}}{\partial \theta^{2}}-\frac{\omega^{2}}{u^{2}}\right) \varphi_{\omega}(\rho, \theta)=-\frac{2 q}{a u \varepsilon(\omega)} \delta(\rho-a) \delta(\theta-\gamma)
$$

The solutions for the electrostatic potential in the regions $0 \leq \theta \leq \alpha$ and $\alpha \leq$ $\theta \leq 2 \pi$ are respectively

$$
\begin{aligned}
\varphi_{\omega}(\rho, \theta)= & \frac{q}{\pi^{2} u} \int_{-\infty}^{+\infty} d \mu K_{i \mu}(k a) K_{i \mu}(k \rho) \times \\
& \times\left\{\begin{array}{l}
\frac{1}{\varepsilon_{1}(\omega)}\left\{\operatorname{ch}[\mu(\pi-|\theta-\gamma|)]+\psi_{1 \omega}(\mu, \theta)\right\}, \quad 0 \leq \theta \leq \alpha \\
\frac{1}{\varepsilon_{2}(\omega)} \psi_{2 \omega}(\mu, \theta), \quad \alpha \leq \theta \leq 2 \pi .
\end{array}\right.
\end{aligned}
$$


where $K_{\nu}(x)$ is a modified Bessel function of order $\nu=i \mu, k=|\omega| / u$,

$$
\begin{aligned}
& \psi_{1 \omega}(\mu, \theta)=A_{\omega}(\mu) \operatorname{ch}(\mu \theta)+B_{\omega}(\mu) \operatorname{sh}(\mu \theta), \\
& \psi_{2 \omega}(\mu, \theta)=C_{\omega}(\mu) \operatorname{ch}(\mu \theta)+D_{\omega}(\mu) \operatorname{sh}(\mu \theta) .
\end{aligned}
$$

The first term in the large brackets of equation (4) corresponds to the potential of the particle in the unbounded medium with dielectric function $\varepsilon_{1}(\omega)$ (particular solution of the inhomogeneous equation (3)). The other terms in equation (4) correspond to the potential generated due to the existence of the interfaces (solution of the homogeneous equation (3) or Laplace's equation (Dobrzynsky and Maradudin 1972)).

The coefficients $A_{\omega}(\mu), B_{\omega}(\mu), C_{\omega}(\mu)$ and $D_{\omega}(\mu)$ are determined by the boundary conditions. The potential must be continuous at $\theta=0$ and $\theta=\alpha$, and the normal component of the Fourier amplitude of the electric displacement vector must be continuous at $\theta=0$ and $\theta=\alpha$. From equation (4) we can obtain the set of following equations for coefficients $A_{\omega}(\mu), B_{\omega}(\mu), C_{\omega}(\mu)$ and $D_{\omega}(\mu)$

$$
\begin{gathered}
\frac{1}{\varepsilon_{1}(\omega)}\left[\operatorname{ch}[\mu(\pi+\gamma-\alpha)]+A_{\omega}(\mu) \operatorname{ch}(\mu \alpha)+B_{\omega}(\mu) \operatorname{sh}(\mu \alpha)\right] \\
=\frac{1}{\varepsilon_{2}(\omega)}\left[C_{\omega}(\mu) \operatorname{ch}(\mu \alpha)+D_{\omega}(\mu) \operatorname{sh}(\mu \alpha)\right] \\
\frac{1}{\varepsilon_{1}(\omega)}\left[\operatorname{ch}[\mu(\pi-\gamma)]+A_{\omega}(\mu)\right]=\frac{1}{\varepsilon_{2}(\omega)}\left[C_{\omega}(\mu) \operatorname{ch}(2 \pi \mu)+D_{\omega}(\mu) \operatorname{sh}(2 \pi \mu)\right] \\
=C_{\omega}(\mu) \operatorname{sh}(\mu \alpha)+D_{\omega}(\mu) \operatorname{ch}(\mu \alpha), \\
\operatorname{sh}[\mu(\pi-\gamma)]+B_{\omega}(\mu)=C_{\omega}(\mu) \operatorname{sh}(2 \pi \mu)+D_{\omega}(\mu) \operatorname{ch}(2 \pi \mu) .
\end{gathered}
$$

Only $A_{\omega}(\mu)$ and $B_{\omega}(\mu)$ are of interest as they represent the coefficients of the homogeneous portion of the potential and thus are needed to obtain the selfenergy and stopping power.

We seek the dissipative component of the force acting on the beam moving near the wedge surface. We neglect quantum recoil effects and assume that $u$ is constant (i.e., the external charge acts as an infinite source of energy and momentum). The negative of the dissipative component of the induced force is the specific energy loss (Ritchie 1957)

$$
-\frac{d W}{d z}=\int_{0}^{\infty} d \omega \hbar \omega P(\omega)
$$


where

$$
P(\omega)=\frac{2 q^{2}}{\pi \hbar u^{2}}\left\{\operatorname{Im} \frac{-1}{\varepsilon_{1}(\omega)} \ln \frac{k_{c} u}{\omega}+\frac{2}{\pi} \int_{0}^{\infty} d \mu K_{i \mu}^{2}\left(\frac{\omega}{u} a\right) \operatorname{sh}[\mu(2 \pi-\alpha)] Q_{\omega}(\mu)\right\}
$$

is the excitation probability,

$$
\begin{gathered}
Q_{\omega}(\mu)=-\operatorname{Im}\left\{\frac{\eta_{s}(\omega)}{\varepsilon_{1}(\omega)} \frac{\eta_{s}(\omega) \operatorname{sh}[\mu(\pi-\alpha)]+\operatorname{sh}(\pi \mu) \operatorname{ch}[\mu(2 \gamma-\alpha)]}{\operatorname{sh}^{2}(\pi \mu)-\eta_{s}^{2}(\omega) \operatorname{sh}^{2}[\mu(\pi-\alpha)]}\right\}, \\
\eta_{s}(\omega)=\frac{\varepsilon_{1}(\omega)-\varepsilon_{2}(\omega)}{\varepsilon_{1}(\omega)+\varepsilon_{2}(\omega)}
\end{gathered}
$$

is the surface response function for plane geometry, $k_{c}=2 m u / \hbar$ is a cutoff wave number (Brandt et al 1974). Note that this definition of $k_{c}$ is valid when charged particle beam is sufficiently fast, i.e. $u>e^{2} / \hbar \simeq 2.2 \times 10^{8} \mathrm{~cm} / \mathrm{sec}$. As expected, the excitation probability in equation (11) contains terms corresponding to both the excitation of the bulk (the term $\operatorname{Im}\left(-1 / \varepsilon_{1}\right)$ ) and of the surface modes of the wedge. Therefore, if one wishes to probe the surface excitation field, without interference from the bulk modes, the probe has to be kept external to the wedge, as was done in the experiments (Marks 1982, Cowley 1982, Wheatley et al 1983). One can calculate the specific energy loss from equations (11) and (12).

The dispersion relation for the surface modes is the result of equating the denominator in equation (12) to zero. As it follows from the expression (12), there are two type of surface modes. The frequency of the first one (so called even mode), in the case of $\varepsilon_{2}(\omega)=1, \varepsilon_{1}(\omega)=\varepsilon(\omega)$, is determined from the dispersion equation:

$$
\varepsilon(\omega)=-\frac{\operatorname{th}\left[\mu\left(\pi-\frac{\alpha}{2}\right)\right]}{\operatorname{th}\left(\frac{\mu \alpha}{2}\right)} .
$$

The electric potential in this surface mode is symmetric as related to the symmetry plane of the wedges (the plane $\theta=\alpha / 2$ ) (Dobrzynsky and Maradudin 1972). The frequency of the second one (so called odd mode) is determined from the dispersion equation:

$$
\varepsilon(\omega)=-\frac{\operatorname{th}\left(\frac{\mu \alpha}{2}\right)}{\operatorname{th}\left[\mu\left(\pi-\frac{\alpha}{2}\right)\right]} .
$$

The electric potential in this type of surface mode is antisymmetric as related to the symmetry plane of the wedges (Dobrzynsky and Maradudin 1972). When $\varepsilon_{2}(\omega)=\varepsilon(\omega), \varepsilon_{1}(\omega)=1$, the above mentioned dispersion equations for the odd and even surface modes change their places. 
We consider first the case of a beam travelling external to the wedge, in vacuum $\left(\varepsilon_{1}(\omega)=1, \varepsilon_{2}(\omega)=\varepsilon(\omega)\right)$. Then from equations (11)-(13) we have

$$
\begin{gathered}
P(\omega)=\frac{4 q^{2}}{\pi^{2} \hbar u^{2}} \int_{0}^{\infty} d \mu K_{i \mu}^{2}\left(\frac{\omega}{u} a\right) \operatorname{sh}\left[\mu(2 \pi-\alpha) Q_{\omega}(\mu),\right. \\
Q_{\omega}(\mu)=\operatorname{Im}\left\{\eta(\omega) \frac{\eta(\omega) \operatorname{sh}[\mu(\alpha-\pi)]-\operatorname{sh}(\pi \mu) \operatorname{ch}[\mu(2 \gamma-\alpha)]}{\eta^{2}(\omega) \operatorname{sh}^{2}[\mu(\alpha-\pi)]-\operatorname{sh}^{2}(\pi \mu)}\right\},
\end{gathered}
$$

where

$$
\eta(\omega)=\frac{\varepsilon(\omega)-1}{\varepsilon(\omega)+1}
$$

We consider now the case of a beam travelling through the wedge, but parallel to the edge. (i.e. $\varepsilon_{2}(\omega)=1, \varepsilon_{1}(\omega)=\varepsilon(\omega)$ )

$$
\begin{gathered}
P(\omega)=\frac{2 q^{2}}{\pi \hbar u^{2}}\left\{\operatorname{Im} \frac{-1}{\varepsilon(\omega)} \ln \frac{k_{c} u}{\omega}+\frac{2}{\pi} \int_{0}^{\infty} d \mu K_{i \mu}^{2}\left(\frac{\omega}{u} a\right) \operatorname{sh}[\mu(2 \pi-\alpha)] Q_{\omega}(\mu)\right\}, \\
Q_{\omega}(\mu)=\operatorname{Im}\left\{\frac{\eta(\omega)}{\varepsilon(\omega)} \frac{\eta(\omega) \operatorname{sh}[\mu(\pi-\alpha)]+\operatorname{sh}(\pi \mu) \operatorname{ch}[\mu(2 \gamma-\alpha)]}{\eta^{2}(\omega) \operatorname{sh}^{2}[\mu(\pi-\alpha)]-\operatorname{sh}^{2}(\pi \mu)}\right\} .
\end{gathered}
$$

Now both terms in equation (19) contribute to the specific energy loss of the beam travelling through the wedge.

It is instructive to derive from equations (16) and (19) the excitation probability quoted by Marks (1982) for an electron beam traveling parallel to a semiinfinite dielectric occuping the region $0 \leq \theta \leq \pi$ (or $\pi \leq \theta \leq 2 \pi$ ), and at a distance $d$ from its surface. Substituting $\alpha=\pi$ in the equations (16) and (19) we find

$$
P(\omega)=\frac{4 q^{2}}{\pi^{2} \hbar u^{2}} \operatorname{Im}[\eta(\omega)] \int_{0}^{\infty} d \mu K_{i \mu}^{2}\left(\frac{\omega}{u} a\right) \operatorname{ch}[\mu(\pi-2 \gamma)],
$$

when $\varepsilon_{1}(\omega)=1, \varepsilon_{2}(\omega)=\varepsilon(\omega)$, and

$$
P(\omega)=\frac{2 q^{2}}{\pi \hbar u^{2}}\left\{\operatorname{Im} \frac{-1}{\varepsilon(\omega)} \ln \frac{k_{c} u}{\omega}-\frac{2}{\pi} \operatorname{Im}\left[\frac{\eta(\omega)}{\varepsilon(\omega)}\right] \int_{0}^{\infty} d \mu K_{i \mu}^{2}\left(\frac{\omega}{u} a\right) \operatorname{ch}[\mu(\pi-2 \gamma)]\right\}
$$

when $\varepsilon_{2}(\omega)=1, \varepsilon_{1}(\omega)=\varepsilon(\omega)$. For calculation of integral in equations (21) and (22) we have used the following expression for modified Bessel function (Bateman and Erdelyi 1977)

$$
K_{i \mu}^{2}(x)=\frac{\pi}{\operatorname{sh}(\pi \mu)} \int_{0}^{\infty} d t \sin (2 \mu t) J_{0}(2 x \operatorname{sh} t)
$$


where $J_{0}(x)$ is the Bessel function of zeroth order. By using equation (23) we finally find

$$
P(\omega)=\frac{2 q^{2}}{\pi \hbar u^{2}} \operatorname{Im}\left[\frac{\varepsilon(\omega)-1}{\varepsilon(\omega)+1}\right] K_{0}\left(2 \frac{\omega}{u} d\right),
$$

when $\varepsilon_{1}(\omega)=1, \varepsilon_{2}(\omega)=\varepsilon(\omega)$, and

$$
P(\omega)=\frac{2 q^{2}}{\pi \hbar u^{2}}\left\{\operatorname{Im} \frac{-1}{\varepsilon(\omega)} \ln \frac{k_{c} u}{\omega}+\operatorname{Im}\left[\frac{1-\varepsilon(\omega)}{\varepsilon(\omega)(1+\varepsilon(\omega))}\right] K_{0}\left(2 \frac{\omega}{u} d\right)\right\},
$$

when $\varepsilon_{2}(\omega)=1, \varepsilon_{1}(\omega)=\varepsilon(\omega), d=a \sin \gamma$ is a distance of particle from surface, $K_{0}$ is the modified Bessel function of zeroth order.

Dobrzynsky and Maradudin (1972) showed that in the limit of large values of $\mu$ or in the case of $\alpha \rightarrow \pi$ the dispersion relation of the edge modes, Eqs (14) and (15), coincides with the dispersion relation $(\varepsilon=-1)$ for surface plasmon modes bound to the plane interface between a dielectric medium and vacuum. Consequently, the surface energy-loss function in equations (16) and (19) reduces to the surface energy-loss function in equations $(24)$ and $(25), \operatorname{Im}[(\varepsilon-1) /(\varepsilon+1)]$, in the limit $\alpha \rightarrow \pi$.

\section{Analysis and comparison with other works}

We have evaluated the excitation probabilities in Eqs (16)-(19) with the complex dielectric function for $\mathrm{MgO}$ taken from experimental data (Roessler and Walker 1967), and for an 80-keV electron beam as in the experiment (Marks 1982).

We first briefly recall the principal results of the investigations carried out by Marks (1982) and Cowley (1982).

(i) The overall intensity in the EELS spectrum (and therefore also the intensity of a given peak) decreases when the electron path goes from a lateral surface to the edge of the crystal (Marks 1982) (see also figures 2-8 below).

(ii) For electron paths along a lateral surface, the intensity of a given peak first slowly increases and then rapidly decreases exponentially as the beam-surface distance goes from inside to outside the wedge (Marks 1982) (see also figure 9 below).

(iii) For electron paths both parallel to the lateral surface and along the edge, a surface plasmon at $18 \mathrm{eV}$ was observed, together with a strong enhancement of the low frequencies (in comparison with the spectrum for electron paths through the bulk, see figure 8 below). The 18-eV peak was attributed by Marks (1982) to a genuine surface resonance, in contrast to Cowley's (1982) interpretation of it as due to transition radiation.

Now we are giving the numerical analyzes of the expressions (16) and (19) for the excitation probability for $M g O$. We have utilized available bulk optical data 
(Roessler and Walker 1967) to calculate the differential energy-loss probability. Figs. 2-6 show the excitation probability for a beam traveling in vacuum parallel to the surface of the sharp-edged wedge at a various wedge interior and beam orientation angles $\alpha^{*}=2 \pi-\alpha$ and $\gamma$, at a distance from edge of $a=2 \mathrm{~nm}$. In Figs. $2-4$ the angle $\alpha^{*}$ is obtuse, meanwhile in figure 5 the angle $\alpha^{*}$ is acute and in this case the dielectric wedge is well-defined. Note that as the angle $\alpha$ increases (the angle $\alpha^{*}$ decreases) and the wedge becomes well-defined, the maximum intensity of excitation probability shifts from high value of $\omega$ to low plasmon energy region. Also the intensity of given peak increases when the electron beam path approaches from the symmetry plane of the wedge to the wedge lateral surface.

Figure 6 shows the excitation probability as a function of wedge interior angle $\alpha$ for $a=2 \mathrm{~nm}$ and $\omega=13.6 \mathrm{eV}$. The solid lines correspond to the cases when beam position angle $\gamma$ is changed with increasing of $\alpha$ (the following four values for angle $\gamma$ are considered: $\gamma=\alpha / 2, \gamma=\alpha / 4, \gamma=\alpha / 6$ and $\gamma=\alpha / 10$ respectively). The dotted and dashed lines correspond to the case when electron beam position angle is fixed with $\gamma=8^{0}$ and $\gamma=\pi / 2$ respectively. From this figure it follows that excitation probability of a given mode in all curves decreases rapidly after the value of $\alpha \simeq 3 \pi / 2 \simeq 4.5 \mathrm{rad}$.

The resonance at $\omega \sim 18 \mathrm{eV}$ in Figs. 3-5 and in solid curve of figure 7 was clearly observed in the experiments (Marks 1982, Cowley 1982), and is not so distinctly apparent in the predictions for the semi-infinite model of the wedge (Garcia-Molina et al 1985, Marks 1982) (see also dotted curve in figure 7). Also this resonance is absent in the case of very large value of the wedge interior angle $\alpha^{*}$ (see figure 2). Cowley (1982) attributed this $\sim 18-\mathrm{eV}$ mode to transition radiation, but its origin as a genuine surface resonance is clear from the model calculations for the parabolically shaped wedge (Garcia-Molina et al 1985) or from the present calculations for sharp-edged wedge.

Figure 7 shows the excitation probability for a beam travelling parallel to the edge of the wedge and in front of it, at a distance of $a=2 \mathrm{~nm}$. The solid curve corresponds to a well-defined wedge $\left(\alpha=11 \pi / 6\right.$ or $\left.\alpha^{*}=\pi / 6\right)$, for an angle $\gamma=11 \pi / 12$, and dotted curve is the prediction for a semi-infinite medium. This latter case, which has been reduced by a factor of 5 , is very similar to the prediction of nearly flat parabolically shaped wedge (Garcia-Molina et al 1985).

For a wedge boundary defined by $\alpha=11 \pi / 6$, figure 8 shows a comparison between the excitation probabilities of the wedge when the electron beam passes in front of the edge $(\gamma=11 \pi / 12)$ (dashed curve, which is the same as solid curve in figure 7) or along one of its lateral surfaces (solid line). In the first case $a=2$ $\mathrm{nm}$, in the second one $a \simeq 20.1 \mathrm{~nm}$ (the beam distance to the edge is taken to be $20 \mathrm{~nm}$ and therefore $\gamma \simeq 0.1$ ). Also shown in figure 8 is the excitation probability (reduced by a factor of 40) for a beam traveling through the bulk of the wedge $(\alpha=\pi / 6)$, along its symmetry plane (dotted line), and at a distance $20 \mathrm{~nm}$ from its edge. The results in figure 8 may be compared with the experimental findings in figure 2 of Marks (1982). The details of the experiment are reproduced by our 
calculations. For instance, in the beam-lateral surface interaction spectrum, the intensity of the $\sim 18-\mathrm{eV}$ peak is greater than the intensity of the $\sim 13$-eV peak. Also, the $\sim 18-\mathrm{eV}$ peak in this spectrum shifts to $\sim 22 \mathrm{eV}$ in the bulk spectrum. The bulk plasmon for $M g O$ is located at $\sim 22 \mathrm{eV}$ (Roessler and Walker 1967), as seen in figure 8. Note that for calculation of bulk energy losses we have used the cutoff wave number $k_{c}=2 m u / \hbar$ (Brandt et al 1974) which is much grater than that of used in other works $\left(k_{c} \sim 0.1 \mathrm{~nm}^{-1}\right.$ ) (Illman et al 1988, Garcia-Molina et al 1985, Marks 1982).

As mentioned in (ii) above, Marks (1982) also investigated the excitation probability of the wedge for electron beam positions ranging from $\sim 10 \mathrm{~nm}$ with respect to the wedge surface, but inside the wedge, up to $\sim 10 \mathrm{~nm}$ outside the wedge surface. The beam path in the experiment was far from the edge of the wedge, and the dimensions of the cubic crystal were $\sim 100 \mathrm{~nm}$. We have evaluated the corresponding expressions, (16) and (19), for the wedge interior angle $\pi / 3$ and for beam distances from edge $\geq 50 \mathrm{~nm}$. The results are shown in figure 9 . In agreement with the experimental results (figure 3 in the work of Marks (1982), see also point (ii) above), the excitation probability decays exponentially with distance, the slope being larger with increasing the energy value $\omega$. The relative intensities of the different curves are also in agreement with the experimental results, the curve for $\omega=10 \mathrm{eV}$ crossing the other curves shown in figure 9 . In the experiment the transition from inside to outside the wedge is broader than figure 9 shows. One should note, however, that our calculations was done for a point-like charged particle beam, whereas the experimental value of the beam size was rather large, $\sim 2 \mathrm{~nm}$. We recall also that the spectrometer resolution in Marks' (1982) experiment is $3 \mathrm{eV}$.

One of the most easily controlled variables affecting the excitation probability is the incident-beam energy. It is clearly of practical interest to determine the optimum incident energy which will elicit the greatest response from a given target. To this end we have determined the energy which would maximize the excitation probability for a given surface mode and a wedge shape. In figure 10 we show the dependence of excitation probability for given surface mode $(\omega=13.6$ $\mathrm{eV})$ as a function of beam kinetic energy $\left(1 \mathrm{keV} \leq E_{\text {kin }} \leq 100 \mathrm{keV}\right)$ for $\alpha=3 \pi / 2$ $\left(\alpha^{*}=\pi / 2\right)$ and for various beam positions (the dotted, dashed, solid and dotdashed lines correspond to $\gamma=3 \pi / 4, \gamma=3 \pi / 8, \gamma=3 \pi / 16$ and $\gamma=3 \pi / 32$ respectively. The latter two have been divided by factors of 2 and 6 respectively). The beam travels in vacuum at a distance $a=2 \mathrm{~nm}$ from the edge. It is evident that the excitation probability first, increases rapidly together with the beam energy and after some value (which grows together with $\gamma$ ) slowly decreases. The plot is given for a 1-100 keV energy range and for $E_{\text {kin }}<1 \mathrm{keV}$, however, our presumption of a rectilinear trajectory becomes questionable, and we have not shown calculations below the $1-\mathrm{keV}$ level. Also for the beam energy range $E_{\text {kin }}>$ $100 \mathrm{keV}$ the retardation effects become important and a separate investigation is required. 


\section{Conclusion}

We have investigated here the case of a beam traveling parallel to the edge of a sharp-edged wedge both in vacuum and through the medium. Other configurations, like beam trajectories at constant $z$, trajectories intersecting the tip of the wedge, or reflecting at the lateral surface, may also be of interest in the analysis of the experiments. Note also that the experiment has been performed with a relatively broad probe, $\sim 2 \mathrm{~nm}$ in diameter, which is comparable to the distance from the beam to the wedge whereas the calculations developed in this paper assume a point-like STEM probe.

We have analyzed the electron-wedge interaction in the electrostatic limit. The electron beam energy $(\sim 100 \mathrm{keV})$ is large enough that one may worry about the effect of retardation on the theoretical predictions. This is currently being investigated.

Finally, let us mention that the expressions derived for the excitation probability in equations (16) and (19) can be used efficiently with moderate computing resources in practical data analysis.

\section{ACKNOWLEDGMENTS}

It is pleasure for authors to thank Professor N.R. Arista for providing with the optical data for a number of materials.

\section{Appendix}

Here we show that in the static limit $(u \rightarrow 0)$ from equations $(2)$ and (4)(9) follows the expression for the potential of a charged particle located near the conducting $(\varepsilon \rightarrow \infty)$ wedge surface. For this we substitute $\omega=\kappa u$ in equation (2) limit of $u \rightarrow 0$. We find the following expression (note that inside the conductor the potential is zero).

$$
\begin{aligned}
\varphi(\rho, \theta, z)= & \frac{2 q}{\pi^{2}} \int_{0}^{\infty} d \kappa \cos (\kappa z) \int_{-\infty}^{+\infty} d \mu K_{i \mu}(\kappa a) K_{i \mu}(\kappa \rho) \times \\
& \times\{\operatorname{ch}[\mu(\pi-|\theta-\gamma|)]+A(\mu) \operatorname{ch}(\mu \theta)+B(\mu) \operatorname{sh}(\mu \theta)\},
\end{aligned}
$$


where $A(\mu)=A_{\omega}(\mu), B(\mu)=B_{\omega}(\mu)$ at $\omega \rightarrow 0$. Taking into account that for conductors $\varepsilon \rightarrow \infty$ when $\omega \rightarrow 0$ (Landau and Lifshitz 1982) from equations (6)-(9) we can obtain the following set of equations

$$
\begin{gathered}
A(\mu)=-\operatorname{ch}[\mu(\pi-\gamma)], \\
A(\mu) \operatorname{ch}(\mu \alpha)+B(\mu) \operatorname{sh}(\mu \alpha)=-\operatorname{ch}[\mu(\pi-\alpha+\gamma)] .
\end{gathered}
$$

By solving the set of equations (A.2) and (A.3) we find

$$
\varphi(\rho, \theta, z)=\frac{4 q}{\pi^{2}} \int_{0}^{\infty} d \kappa \cos (\kappa z) \int_{-\infty}^{+\infty} d \mu K_{i \mu}(\kappa a) K_{i \mu}(\kappa \rho) C(\mu),
$$

where

$$
C(\mu)=\frac{\operatorname{sh}(\pi \mu)}{\operatorname{ch}(\alpha \mu)} \begin{cases}\operatorname{sh}(\mu \gamma) \operatorname{sh}[\mu(\alpha-\theta)] ; & \gamma \leq \theta \leq \alpha \\ \operatorname{sh}[\mu(\alpha-\gamma)] \operatorname{sh}(\mu \theta) ; & \theta \leq \gamma .\end{cases}
$$

Calculating in equation (A.4) the integral by $\kappa$ (Gradshteyn and Ryzhik 1980) we obtain the following expression for potential

$$
\begin{aligned}
\varphi(\rho, \theta, z)= & \frac{q}{\sqrt{a \rho}} \int_{-\infty}^{+\infty} d \mu \frac{\operatorname{th}(\pi \mu)}{\operatorname{sh}(\mu \alpha)} P_{i \mu-1 / 2}(\operatorname{ch} \eta) \\
& \begin{cases}\operatorname{sh}(\mu \gamma) \operatorname{sh}[\mu(\alpha-\theta)] ; & \gamma \leq \theta \leq \alpha \\
\operatorname{sh}[\mu(\alpha-\gamma)] \operatorname{sh}(\mu \theta) ; & \theta \leq \gamma,\end{cases}
\end{aligned}
$$

where $P_{\nu}(x)$ is the Legendre function of the first kind and with order $\nu=i \mu-1 / 2$,

$$
\operatorname{ch} \eta=\frac{\rho^{2}+a^{2}+z^{2}}{2 a \rho} .
$$

By using the known equation for the Legendre function of the second kind $Q_{\nu}(x)$ (Gradshteyn and Ryzhik 1980),

$$
Q_{-i \mu-1 / 2}(z)-Q_{i \mu-1 / 2}(z)=\pi i \operatorname{th}(\pi \mu) P_{i \mu-1 / 2}(z),
$$

we can obtain

$$
\varphi(\rho, \theta, z)=\frac{q}{\pi i \sqrt{a \rho}} \int_{-\infty}^{+\infty} \frac{d \mu}{\operatorname{sh}(\mu \alpha)} Q_{-i \mu-1 / 2}(\operatorname{ch} \eta)\left\{\begin{array}{l}
\operatorname{sh}(\mu \gamma) \operatorname{sh}[\mu(\alpha-\theta)] ; \gamma \leq \theta \leq \alpha \\
\operatorname{sh}[\mu(\alpha-\gamma)] \operatorname{sh}(\mu \theta) ; \theta \leq \gamma .
\end{array}\right.
$$

It is convenient to use the integral presentation of Legendre function of the second kind (Gradshteyn and Ryzhik 1980)

$$
Q_{-i \mu-1 / 2}(\operatorname{ch} \eta)=\int_{\eta}^{\infty} \frac{d \zeta \exp (i \zeta \mu)}{\sqrt{2(\operatorname{ch} \zeta-\operatorname{ch} \eta)}}
$$


By using (A.10) the expression (A.9) can be written as

$$
\varphi(\rho, \theta, z)=\frac{q}{\alpha \sqrt{2 a \rho}} \int_{\eta}^{\infty} \frac{d \zeta \Phi(\zeta, \theta)}{\sqrt{\operatorname{ch} \zeta-\operatorname{ch} \eta}}
$$

where

$$
\Phi(\zeta, \theta)=2 \sum_{n=1}^{\infty} \exp \left(-\frac{\pi \zeta n}{\alpha}\right)\left[\cos \left(\pi n \frac{\gamma-\theta}{\alpha}\right)-\cos \left(\pi n \frac{\gamma+\theta}{\alpha}\right)\right] .
$$

Finally, taking into account the expression (Gradshteyn and Ryzhik 1980)

$$
2 \sum_{n=1}^{\infty} \exp (-n \alpha) \cos (n \beta)=-1+\frac{\operatorname{sh}(\alpha)}{\operatorname{ch}(\alpha)-\cos (\beta)}
$$

from (A.11)-(A.13) we can find the following expression for the potential given by Landau and Lifshitz (1982)

$$
\begin{aligned}
\varphi(\rho, \theta, z)= & \frac{q}{\alpha \sqrt{2 a \rho}} \int_{\eta}^{\infty} \frac{d \zeta \operatorname{sh}\left(\frac{\pi \zeta}{\alpha}\right)}{\sqrt{\operatorname{ch} \zeta-\operatorname{ch} \eta}} \times \\
& \times\left\{\frac{1}{\operatorname{ch}\left(\frac{\pi \zeta}{\alpha}\right)-\cos \left[\frac{\pi(\theta-\gamma)}{\alpha}\right]}-\frac{1}{\operatorname{ch}\left(\frac{\pi \zeta}{\alpha}\right)-\cos \left[\frac{\pi(\theta+\gamma)}{\alpha}\right]}\right\} .
\end{aligned}
$$

\section{References}

Bateman H and Erdelyi A 1977 Higher Transcendental Functions (Moscow: Nauka)

Brandt W, Ratkowski A and Ritchie R H 1974 Phys. Rev. Lett. 331325

Chen C H, Joy D C, Chen H S and Hauser J J 1986 Phys. Rev. Lett. 57743

Cowley J M 1982 Surf. Sci. 114587

1982 Phys. Rev. B 251401

Davis L D 1976 Phys. Rev. B 145523

Dobrzynsky L and Maradudin A A 1972 Phys. Rev. B 63810

Echenique P M, Howie A and Wheatley D J 1987 Philos. Mag. B 56335

Echenique P M and Pendry J B 1975 J. Phys. C 82936

Ferrel T L and Echenique P M 1985 Phys. Rev. Lett. 551526

Garcia-Molina R, Gras Marti A and Ritchie R H 1985 Phys. Rev. B 31121

Gradshteyn I S and Ryzhik I M 1980 Table of Integrals, Series and Products (New York: Academic) 
Illman B L, Anderson V E, Warmack R J and Ferrel T L 1988 Phys. Rev. B 383045

Landau L D and Lifshitz E M 1982 Eloctrodynamics of Continuous Media (Moscow: Nauka)

Marks L D 1982 Solid State Commun. 43727

Nersisyan H B and Hovhannisyan A V (unpublished)

Ritchie R H 1957 Phys. Rev. 106874

Rivacoba A, Zabala N and Echenique P M 1992 Phys. Rev. Lett. 693362

Roessler D M and Walker W C 1967 Phys. Rev. 159733

Smythe W R 1969 Static and Dynamic Electrisity (New York: McGraw-Hill)

Walls M G and Howie A 1989 Ultramicroscopy 2840

Walsh C A 1989 Philos. Mag. 59227

Wheatley D I, Howie A and McMullan D 1983 EMAG Conference Surrey (unpublished)

Zabala N, Rivacoba A and Echenique P M 1989 Surf. Sci. 209465 


\section{Figure Captions}

Figure 1. Dielectric wedges filling the space $-\infty<z<\infty, 0<\theta<\alpha$, $\alpha<\theta<2 \pi$, and characterized by an isotropic dielectric functions $\varepsilon_{1}(\omega)$ and $\varepsilon_{2}(\omega)$ respectively.

Figure 2. Excitation probability of surface modes, equation (16), for electron beam traveling in the vacuum parallel to the surface of the wedge, at a distance of $a=2 \mathrm{~nm}$ from edge. The interior angle of the wedge is obtuse ( $\alpha=\pi / 4$ or $\left.\alpha^{*}=7 \pi / 4\right)$. The solid, dashed and dotted lines correspond to the three positions of the beam $\gamma=\pi / 24, \gamma=\pi / 12$ and $\gamma=\pi / 8$ respectively. The electron beam energy is $80 \mathrm{keV}$.

Figure 3. Same as in figure 2, but here $\alpha=3 \pi / 4$ (or $\alpha^{*}=5 \pi / 4$ ). The solid, dashed and dotted lines correspond to the three positions of the beam $\gamma=\pi / 8$, $\gamma=\pi / 4$ and $\gamma=3 \pi / 8$ respectively.

Figure 4. Same as in figure 2, but here $\alpha=5 \pi / 4$ (or $\alpha^{*}=3 \pi / 4$ ). The solid, dashed and dotted lines correspond to the three positions of the beam $\gamma=5 \pi / 24$, $\gamma=5 \pi / 12$ and $\gamma=5 \pi / 8$ respectively.

Figure 5. Same as in figure 2, but here $\alpha=7 \pi / 4$ (or $\alpha^{*}=\pi / 4$, i.e. the wedge is acute-angled and well-defined). The solid, dashed and dotted lines correspond to the three positions of the beam $\gamma=7 \pi / 24, \gamma=7 \pi / 12$ and $\gamma=$ $7 \pi / 8$ respectively.

Figure 6. Excitation probability of given surface mode $\omega=13.6 \mathrm{eV}$ as a function of $\alpha$ (in radian) for electron beam traveling in the vacuum parallel to the surface of the wedge at a distance of $a=2 \mathrm{~nm}$ from edge. The solid lines correspond to the varying beam position angles $(\gamma=\alpha / 2, \gamma=\alpha / 4, \gamma=\alpha / 6$ and $\gamma=\alpha / 10$ respectively). The dotted and dashed lines correspond to the fixed values of beam position angles $\gamma=8^{0}$ and $\gamma=\pi / 2$ respectively. The electron beam energy is $80 \mathrm{keV}$.

Figure 7. Excitation probability of surface modes, equation (16), for electron beam traveling in the vacuum parallel to the surface of the wedge and in front of it, at a distance of $a=2 \mathrm{~nm}$ from edge. The solid line corresponds to the welldefined wedge $\left(\alpha=11 \pi / 6\right.$ or $\left.\alpha^{*}=\pi / 6\right)$ with the beam position angle $\gamma=11 \pi / 12$, the dotted line corresponds to the semi-infinite wedge $(\alpha=\pi, \gamma=\pi / 2)$. In the latest case the probability has been divided by a factor of 5 . The electron beam energy is $80 \mathrm{keV}$.

Figure 8. Excitation probabilities of surface and bulk modes, equations (16) and (19), for electron beam traveling in the vacuum along the edge of the welldefined wedge $\left(\alpha=11 \pi / 6\right.$ or $\left.\alpha^{*}=\pi / 6\right)$, at a distance of $2 \mathrm{~nm}$ from the edge and with angular position $\gamma=11 \pi / 12$ (dashed line). This curve is same as the solid curve in figure 7; along a lateral surface, at a distance of $2 \mathrm{~nm}$ from it and in this case $\alpha=11 \pi / 6\left(\alpha^{*}=\pi / 6\right), a \simeq 20.1 \mathrm{~nm}(\gamma \simeq 0.1)$ (solid line); and dotted line through the bulk of the wedge (equation (19)), along the symmetry plane $(\alpha=\pi / 6, \gamma=\pi / 12)$ and at a distance of $a=20 \mathrm{~nm}$ from the edge. The 
spectrum in dotted curve has been divided by a factor of 40 . The electron beam energy is $80 \mathrm{keV}$.

Figure 9. Excitation probability, for given modes $\omega$ (in eV), equations (16) and (19), for electron beam paths at a varying distance $D$ (in $\mathrm{nm}$ ) from the lateral surface of the wedge. The beam paths are far away from the edge $(\geq 50 \mathrm{~nm})$, and range from inside $(D<0)$ to outside $(D>0)$ the wedge. Wedge interior angle in both cases is $\pi / 3$. The electron beam energy is $80 \mathrm{keV}$.

Figure 10. Excitation probability for given surface mode $\omega=13.6 \mathrm{eV}$ as a function of electron beam kinetic energy $E_{\text {kin }}\left(1 \mathrm{keV} \leq E_{\text {kin }} \leq 100 \mathrm{keV}\right)$. The beam moves in the vacuum parallel to the surface of the well-defined wedge $(\alpha=3 \pi / 2$ or $\alpha^{*}=\pi / 2$ ), at a distance of $2 \mathrm{~nm}$ from the edge. The dotted, dashed, solid and dot-dashed lines correspond to $\gamma=3 \pi / 4, \gamma=3 \pi / 8, \gamma=3 \pi / 16$ and $\gamma=3 \pi / 32$ respectively. The probabilities in the curves with $\gamma=3 \pi / 16$ and $\gamma=3 \pi / 32$ have been divided by factors of 2 and 6 respectively. 


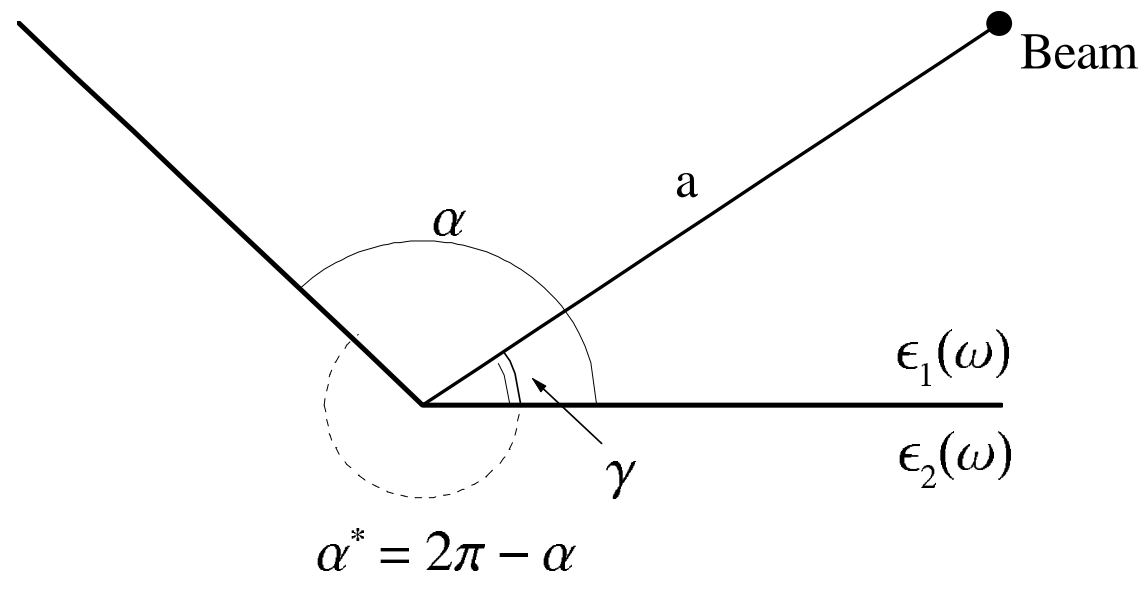

Figure 1 


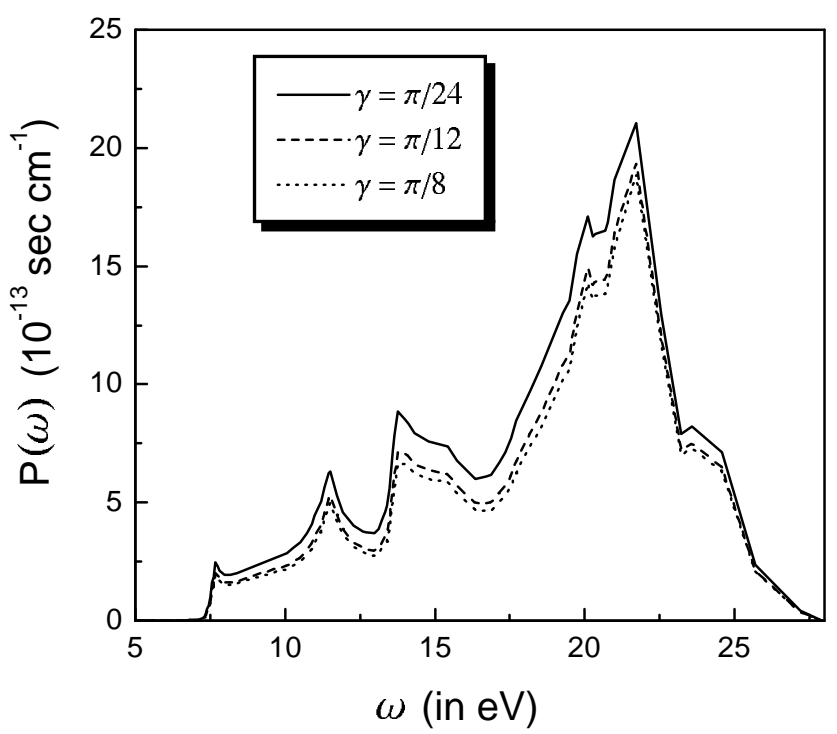

Figure 2 


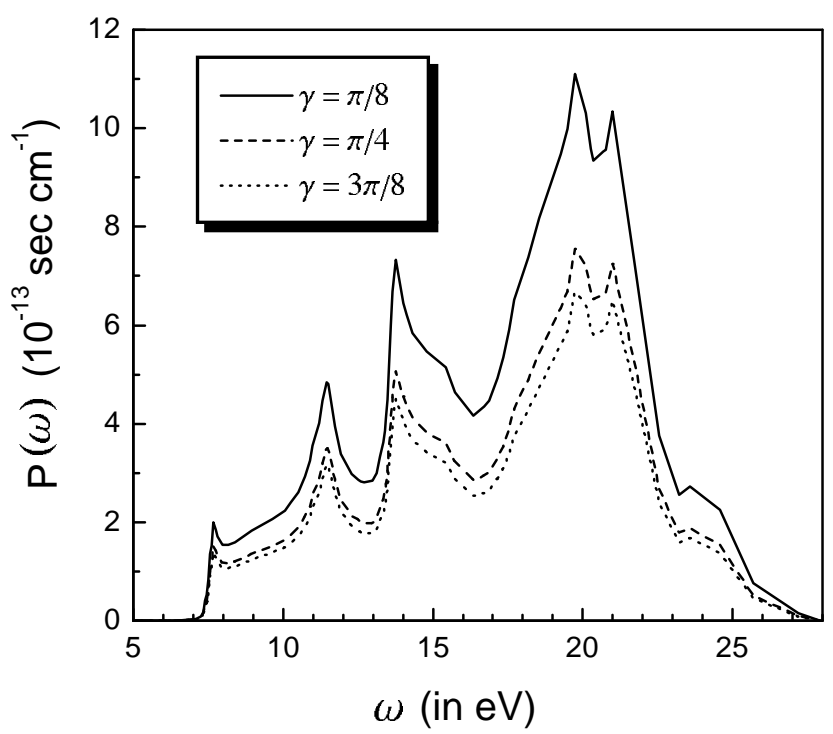

Figure 3 


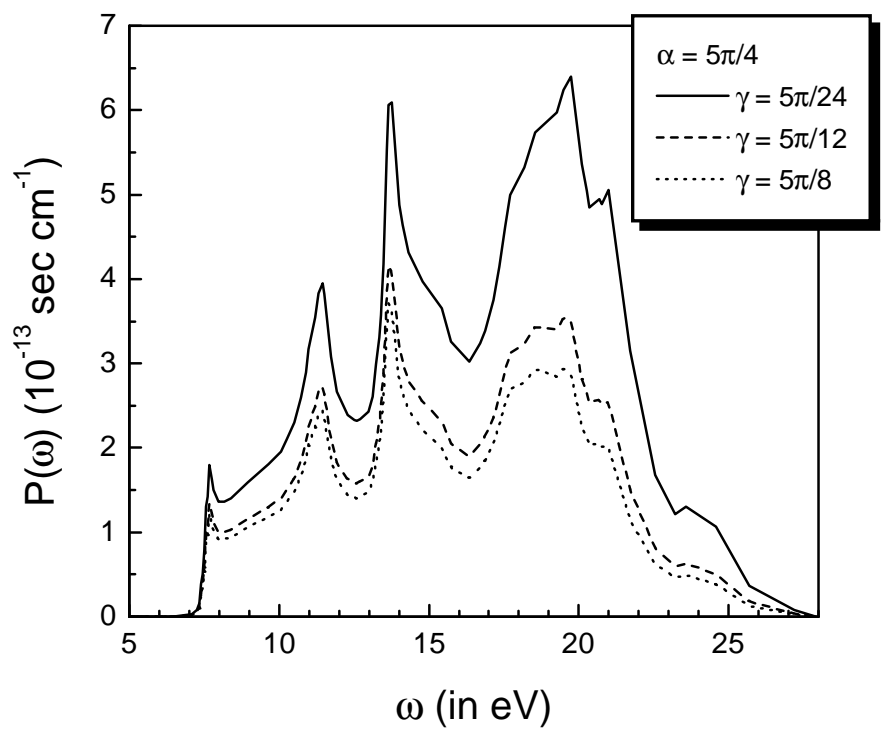

Figure 4 


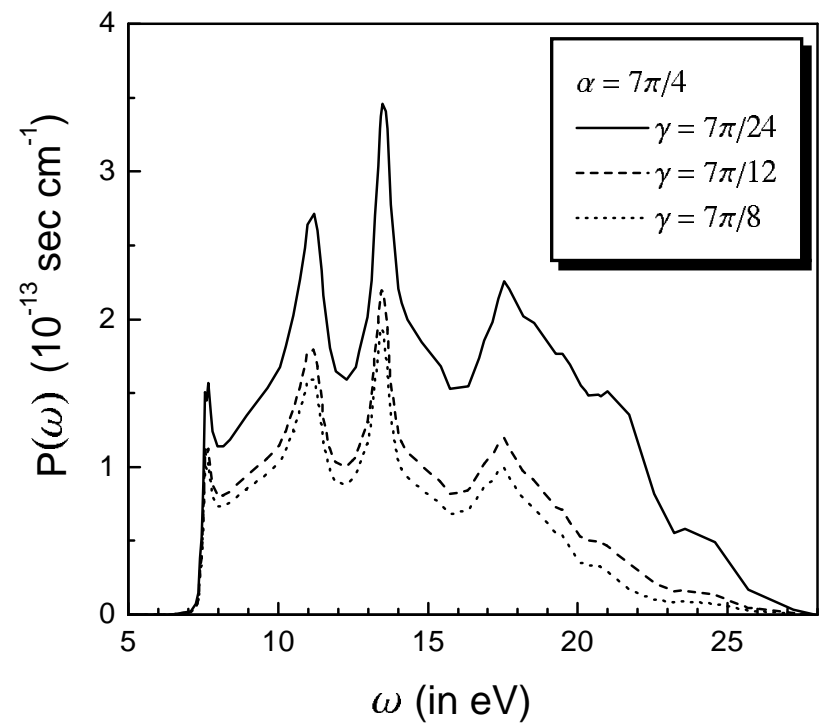

Figure 5 


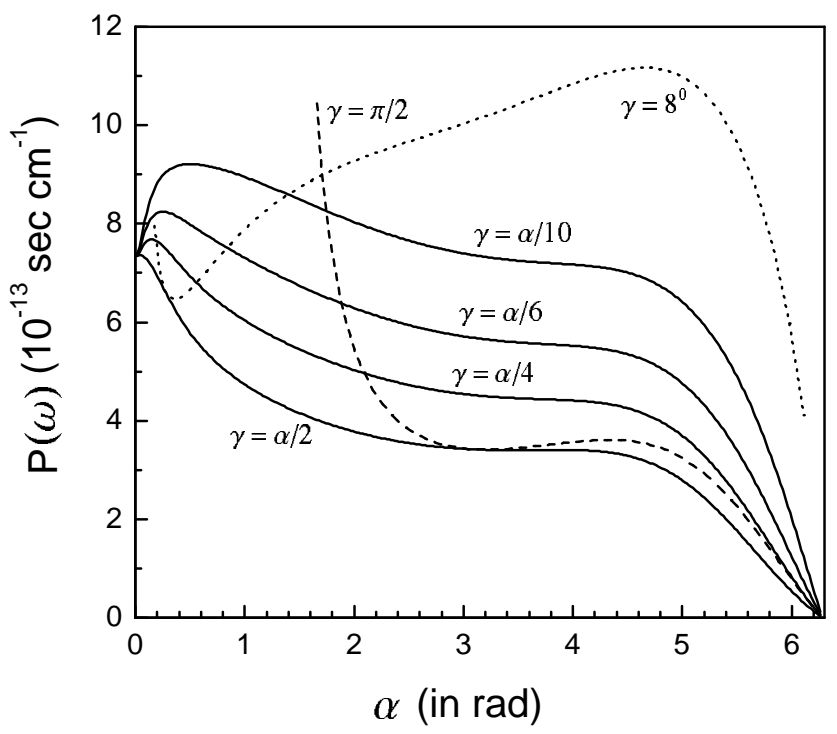

Figure 6 


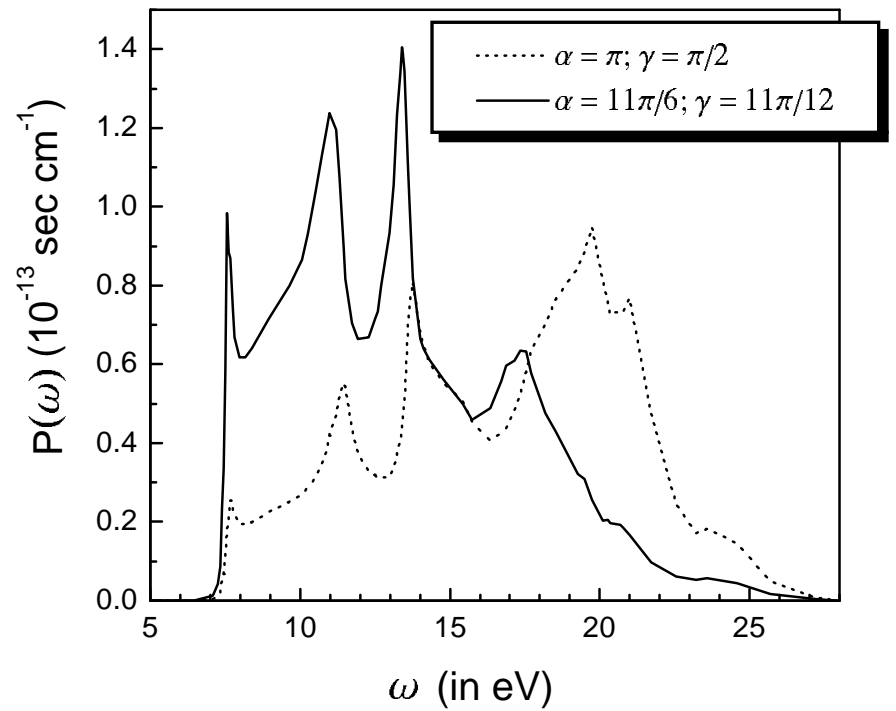

Figure 7 


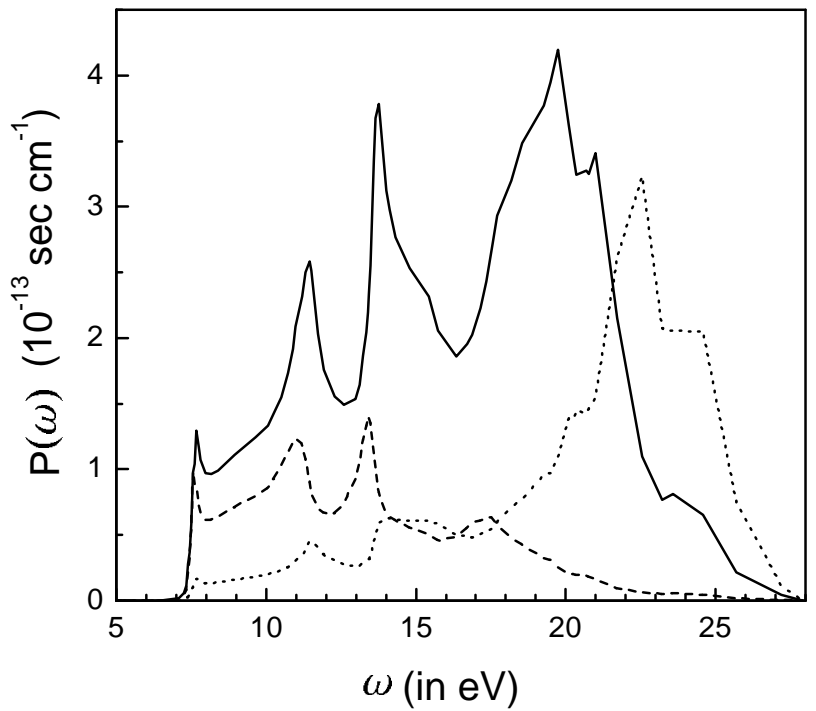

Figure 8 


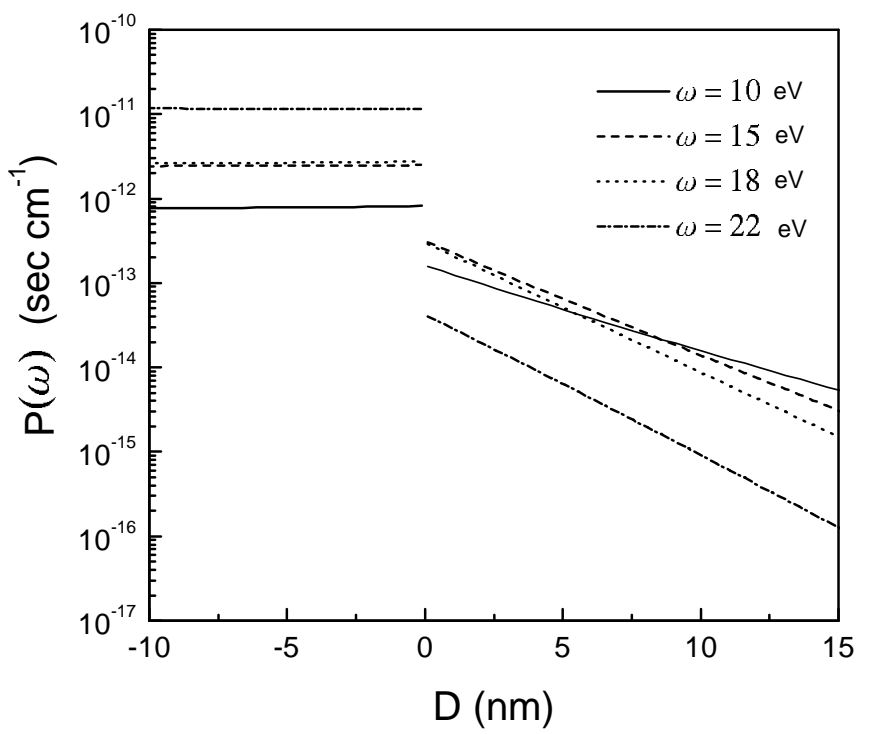

Figure 9 


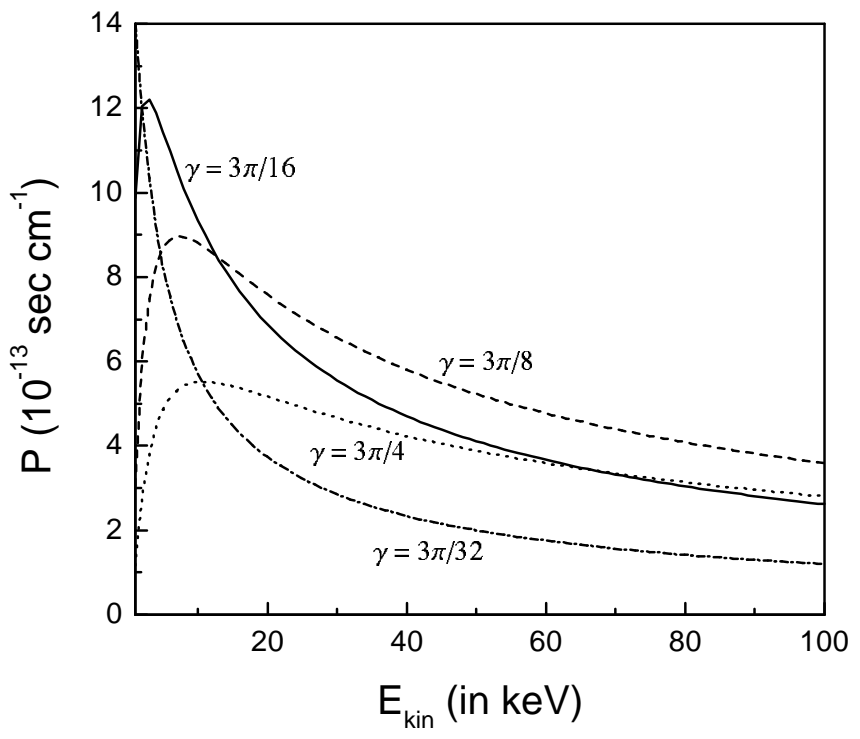

Figure 10 